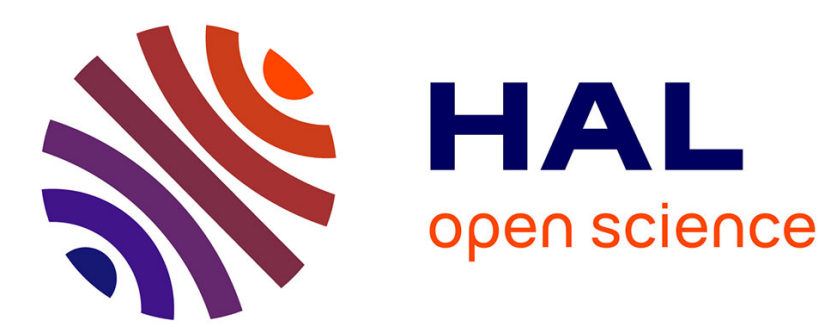

\title{
Etude d'une interface carbone-silicium dans un ruban de silicium polycristallin
}

\author{
J. Goma, M. Oberlin, A. Oberlin
}

\section{To cite this version:}

J. Goma, M. Oberlin, A. Oberlin. Etude d'une interface carbone-silicium dans un ruban de silicium polycristallin. Revue de Physique Appliquée, 1980, 15 (2), pp.229-232. 10.1051/rphysap:01980001502022900 . jpa-00244719

HAL Id: jpa-00244719

https://hal.science/jpa-00244719

Submitted on 1 Jan 1980

HAL is a multi-disciplinary open access archive for the deposit and dissemination of scientific research documents, whether they are published or not. The documents may come from teaching and research institutions in France or abroad, or from public or private research centers.
L'archive ouverte pluridisciplinaire HAL, est destinée au dépôt et à la diffusion de documents scientifiques de niveau recherche, publiés ou non, émanant des établissements d'enseignement et de recherche français ou étrangers, des laboratoires publics ou privés. 


\title{
Etude d'une interface carbone-silicium dans un ruban de silicium polycristallin
}

\author{
J. Goma, M. Oberlin et A. Oberlin \\ Laboratoire Marcel Mathieu, ER 131 du C.N.R.S., UER Sciences, \\ Université d'Orléans, Château de la Source, 45046 Orléans Cedex, France \\ (Reçu le 27 juillet 1979, révisé le 1er octobre 1979, accepté le 2 octobre 1979)
}

\begin{abstract}
Résumé. - Lorsque du silicium se solidifie sur une surface de carbone (pyrocarbone), le carbure de silicium ne forme pas une couche continue entre ces deux phases. Il existe une orientation cristallographique simple entre $\mathrm{Si}$ et $\mathrm{SiC}:(001)_{\mathrm{SiC}}$ parallèle à $(111)_{\mathrm{Si}}$ avec $[100]_{\mathrm{SiC}}$ parallèle à $[011]_{\mathrm{Si}}$. On pense que dans ce cas SiC s'est formé à partir de carbone en solution dans le silicium. Ce carbone proviendrait de l'oxyde de carbone CO présent dans l'atmosphère des fours.
\end{abstract}

Abstract. - When silicon solidifies on a carbon surface (pyrocarbon), the silicon carbide does not form a continuous layer between the two phases. A simple crystallographic orientation exists between $\mathrm{Si}$ and $\mathrm{SiC}:(001)_{\mathrm{SiC}}$ is parallel to $(111)_{\mathrm{Si}},[100]_{\mathrm{Si}}$ being parallel to $[011]_{\mathrm{Si}}$. SiC is thought to have formed from the carbon in solution in silicon. The carbon in solution is supposed to result from the carbon oxide $\mathrm{CO}$ which exists in furnaces.

1. Introduction. - Le laboratoire de Physique et d'Electronique Appliquée (L.E.P.) a mis au point un procédé d'étirage en continu de rubans de silicium polycristallin. Ces rubans sont obtenus par mouillage d'une bande de graphite souple par du silicium liquide [1-4]. Deux procédés sont en développement. Dans le premier, quatre ou deux bandes de graphite souple viennent lécher une zone maintenue fondue au sommet d'un barreau parallélépipédique de silicium chauffé par induction. Dans ce cas, le silicium se dépose sur une face du ruban. Le deuxième procédé consiste à faire passer le ruban de graphite souple au travers d'un creuset rempli de silicium maintenu en fusion. A cet effet, une fente a été ménagée au fond du creuset. On obtient dans ce cas des rubans recouverts de silicium sur les deux faces. Dans les deux cas, le dépôt de silicium est réalisé sous atmosphère d'argon.

Ces procédés sont prometteurs car ils permettraient de produire, à relativement bon marché, du silicium polycristallin en lames minces; malheureusement plusieurs obstacles, tous inhérents au substrat de graphite, freinent actuellement le développement de ces procédés.

Le substrat est un matériau obtenu par laminage de graphite naturel expansé à l'acide sulfurique, commercialisé par la société Le Carbone Lorraine sous le nom de Papyex. Utilisé tel que, il réagit rapidement avec le silicium pour donner du carbure. La réactivité du matériau est due à sa rugosité et à sa porosité. En le protégeant par un dépôt étanche de pyrocarbone obtenu par craquage de méthane à haute température, on peut diminuer la réactivité par un facteur de l'ordre de cent. Cependant, même dans ces conditions, la formation d'une couche de carbure de silicium peut diminuer considérablement le rendement photovoltaïque.

2. Echantillons étudiés. - Nous avons étudié des échantillons obtenus par léchage tangentiel d'une calotte de silicium maintenue fondue au sommet d'un barreau monocristallin. Le substrat était constitué dans ce cas de rubans de papyex recouverts d'une couche de pyrocarbone anisotrope ayant une épaisseur de l'ordre du micron. On a aminci des lames taillées perpendiculairement à l'interface silicium-carbone et on les a assemblées latéralement par deux ou trois dans une résine époxy de façon à avoir plusieurs plans de l'interface soumis à l'amincissement. Deux techniques ont été utilisées : l'ultramicrotome et l'amincissement ionique. Un ultramicrotome muni d'un couteau de diamant a donné de bons résultats pour l'étude du support carboné (papyex et pyrocarbone). Dans le cas de la présence des trois phases : $\mathrm{C}, \mathrm{Si}$ et $\mathrm{SiC}$, les différences de dureté amènent un déchaussement des grains de $\mathrm{Si}$ et de SiC. L'amincissement ionique donne, dans ce cas, d'excellents résultats sur le silicium et le carbure, mais le carbone est par contre 
moins bien abrasé. L'abrasion ionique creuse un cratère dans la plaquette et la zone observable sur les bords du trou correspond à un monocristal de silicium dont une famille de plans (111) est perpendiculaire à la direction moyenne du ruban carboné. Cette relation d'orientation ne provient pas de celle des couches graphitiques dont la désorientation est trop importante mais plus vraisemblablement de la surface des nappes isothermes lors de la cristallisation du silicium.

En effet, une étude des conditions de croissance cristalline du silicium a montré que la germination initiale a lieu à l'interface argon-silicium. Ceci s'explique par la mauvaise conductibilité thermique transversale du papyex qui maintient le silicium en fusion à son contact. C'est donc la direction de l'interface argon-silicium qui imposerait l'orientation cristalline du silicium.

3. Résultats. - Différentes zones du cratère ont été observées d'abord en fond clair (morphologie). Chaque région a été étudiée ensuite par microdiffraction électronique. Enfin chacune des réflexions du diagramme a été isolée par un diaphragme d'ouverture convenable de façon à faire une image agrandie à l'aide du faisceau correspondant (fond noir). On sait que cette technique, couramment utilisée par les métallurgistes, consiste à former une image à partir d'un seul faisceau diffracté. Dans l'image, les régions d'où proviennent le faisceau diffracté apparaissent brillantes sur un champ sombre d'où la dénomination de fond noir.

1) Le papyex est du graphite poreux

$$
\text { (pores } \geqslant 1000 \AA \text { ) }
$$

et formé de cristaux minces et très distordus.

2) Le pyrocarbone est lamellaire et compact (Fig. 2). Toutes les couches aromatiques y sont orientées parallèlement au plan du ruban (plan de dépôt) avec une fluctuation d'orientation prononcée $\left( \pm 25^{\circ}\right)$. Certaines décohésions apparaissent par places (flèches sur la figure 2). La face opposée au papyex est absolument lisse (sans rugosités ni corrosion). Elle apparaît donc intacte et ne semble pas être entrée en réaction avec le silicium, cependant $\mathrm{SiC}$ apparaît.

Le schéma de la figure 1 représente l'un des aspects de la préparation où l'on distingue les trois phases en présence : silicium, carbure de silicium et carbone. On a représenté en encart les diagrammes de diffraction correspondant à deux régions voisines : le pyrocarbone et un cristal de SiC d'une part et le silicium d'autre part. Les clichés de fond noir correspondant aux réflexions encerclées $\mathrm{A}, \mathrm{B}$ et $\mathrm{C}$ sont les figures 2,3 et 4 . Sur le premier (fond noir $00.2 \mathrm{du}$ carbone) on trouve le pyrocarbone sous forme d'une bande lumineuse car les couches aromatiques y sont vues sur la tranche, c'est-à-dire sous l'angle de Bragg 002. Sur le second (fond noir $11 \overline{1}$ du silicium),

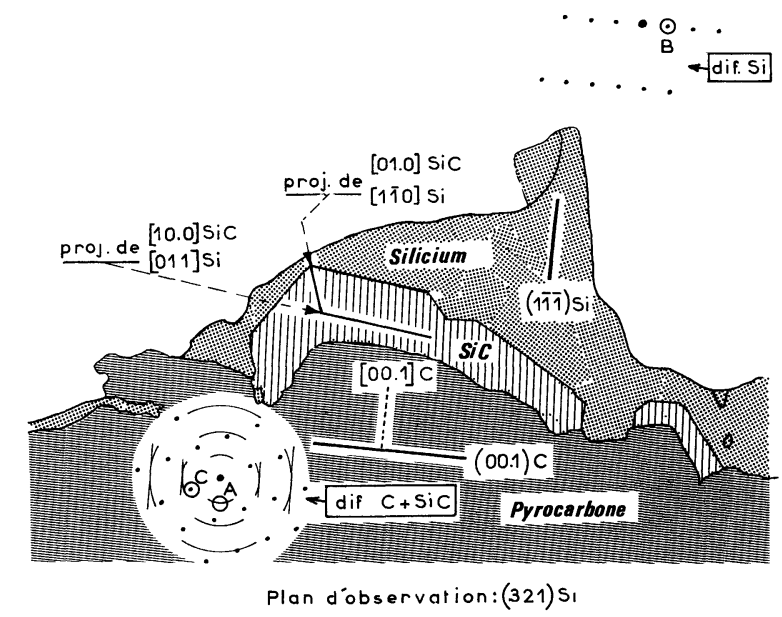

Fig. 1. - Schéma de la préparation obtenue par abrasion ionique ; orientations et diagrammes de diffraction des trois phases en présence.

[Schematic representation of ion beam thinned preparation showing patterns of the three existing phases.]

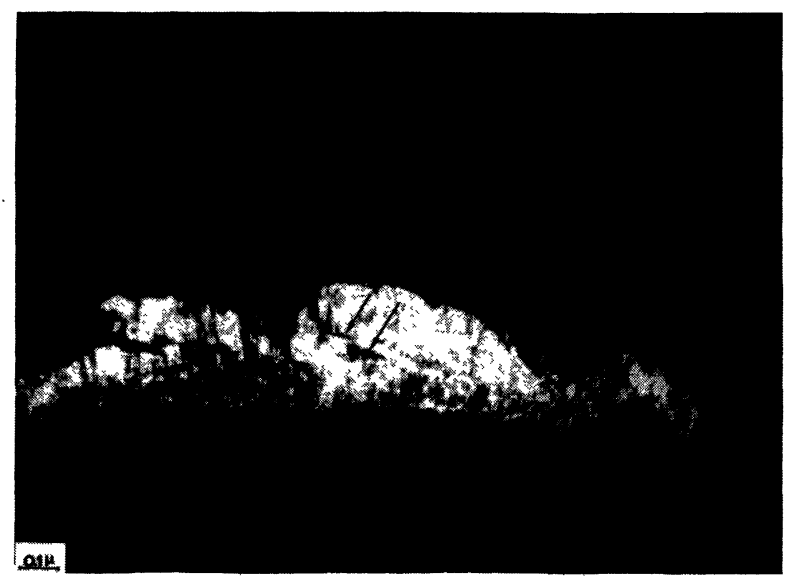

Fig. 2. - Fond noir (002) du pyrocarbone.

[(002) dark-field of pyrocarbon.]

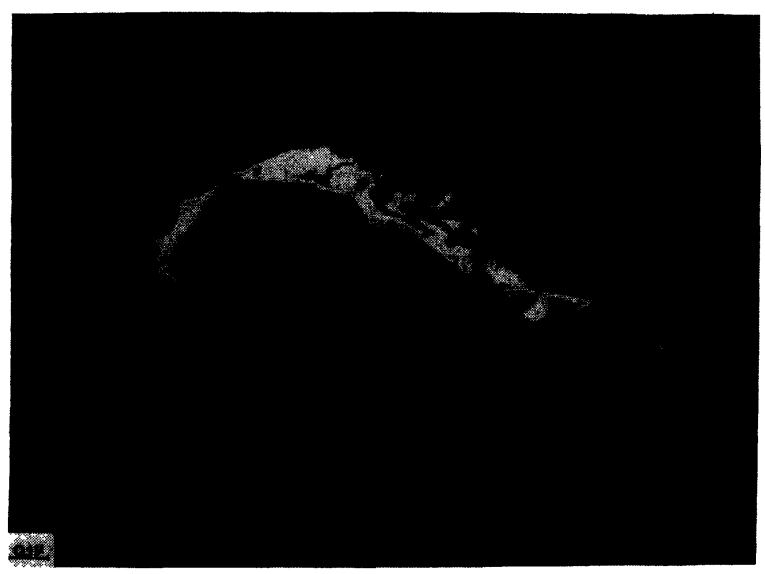

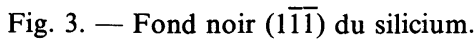

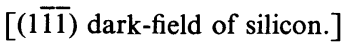




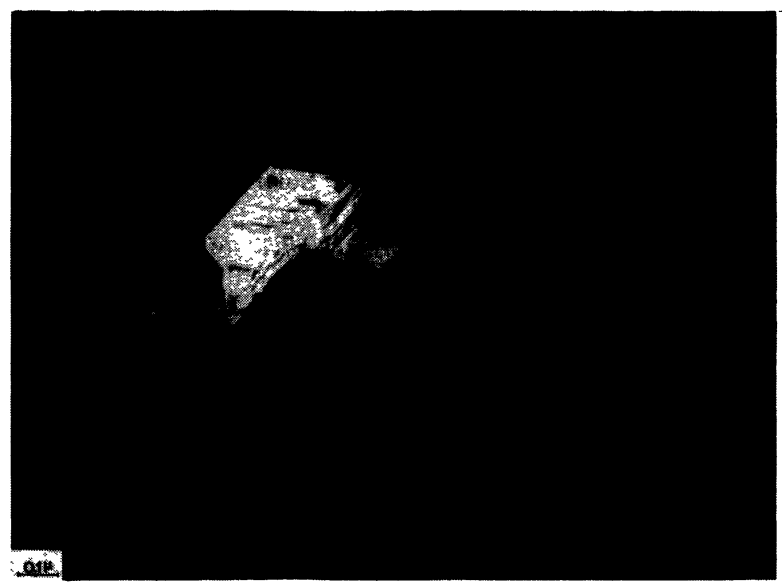

Fig. 4. - Fond noir (100) du carbure de silicium (polytype).

[(100) dark-field of silicon (polytype).]

c'est le silicium qui s'éclaire et montre un réseau de franges d'égale épaisseur dues au biseau formé par l'attaque ionique. Sur le troisième, c'est une des réflexions de $\mathrm{SiC}$ qui est utilisée et le cristal correspondant s'illumine. On voit que le $\mathrm{SiC}$ ne forme pas de couche continue et que le silicium peut être directement en contact avec le carbone sans réagir.

En indexant le diagramme de diffraction électronique du silicium, on voit que le plan d'observation en microscopie électronique est (321) du silicium ce qui permet d'indiquer sur la figure 1 la direction de

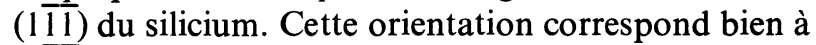
$(111)_{\text {Si }}$ parallèle à $[00.1]_{\mathrm{C}}$. Elle a pour conséquence d'orienter la face $(111)_{\mathrm{Si}}$ obliquement par rapport à $(00.1)_{C}$ et au plan d'observation.

Le carbure se présente en plaquettes hexagonales aplaties d'environ un demi-micromètre dont le diagramme de diffraction n'est interprétable que :

- si on admet qu'il s'agit de $\mathrm{SiC} \alpha$, c'est-à-dire d'un polytype,

- si on admet que le plan (00.1) de ce polytype est confondu avec $(111)_{\mathrm{Si}}$.

Dans ce cas (voir Fig. 1), la rangée [10.0] du polytype est parallèle à $[011]_{\mathrm{Si}}$ tandis que [01.0] du polytype est parallèle à $[1 \overline{10}]_{\mathrm{Si}}$. En même temps, on vérifie que $[01.0]_{\mathrm{SiC}}$ fait $10^{\circ} 49^{\prime}$ avec $[00.1]_{\mathrm{C}}$. La figure 4 en fond noir, où le cristal de $\mathrm{SiC}$ est lumineux, montre que $[10.0]_{\mathrm{SiC}}$ est parallèle aux côtés des plaquettes hexagonales.

Comme la plaquette est oblique par rapport au plan d'observation $(321)_{\mathrm{Si}}$, on observe une déformation du diagramme de $\mathrm{SiC}$ en hexagone irrégulier où les réflexions visibles sont toutes des réflexions $h k l_{\mathrm{SiC} \alpha}$.

Dans tout l'échantillon étudié, on voit qu'il existe une relation d'orientation rigoureuse entre le cristal de silicium et les cristaux de carbure. Cette relation est très simple : le plan (00.1) de $\mathrm{SiC}$ est parallèle au plan (111) de Si. Autrement dit, les feuillets de silicium existant dans le carbure sont parallèles aux plans (111) du Si. On se souvient que le carbure de silicium a une structure comparable à celle de la blende, c'est-à-dire une alternance de plans carbonés situés au quart de la distance entre plans silicium ; l'existence de polytypes n'introduisant que des fautes d'empilement périodiques dans cet ensemble.

Ceci amène à la conclusion logique suivante : si le carbure s'était formé au détriment du pyrocarbone, on devrait trouver $(00.1)_{\mathrm{SiC}}$ parallèle à $(00.1)_{\mathrm{C}}$. Ceci n'a pas été rencontré dans l'échantillon étudié. Par contre, l'orientation entre $(111)_{\mathrm{Si}}$ et $(00.1)_{\mathrm{SiC}}$ tend à montrer que $\mathrm{SiC}$ s'est formé à partir de carbone en solution. L'apparence non altérée du pyrocarbone et l'existence d'interfaces pyrocarbone-silicium sans couche de carbure tend à suggérer la même conclusion.

Une autre expérience nous a montré que la carburation du silicium peut avoir lieu indépendamment du substrat. Dans cette étude, nous avons déposé des petits cristaux de silicium sur des couches très minces de carbone, ayant 50 à $100 \AA$ d'épaisseur. Cet ensemble, supporté par une grille en carbone, a été chauffé dans un four, sous atmosphère d'argon.

Dès $1200^{\circ}$, la transformation du silicium en carbure est presque totale. Quelle que soit la nature du film substrat en carbone (c'est-à-dire graphité ou non) on assiste à une carburation sans aucune corrosion du substrat à des températures inférieures à $1400^{\circ}$. Même si l'on tente de faire fondre les cristaux de silicium en portant très rapidement le substrat à $1450^{\circ}$, on observe une carburation du silicium à l'état solide sans attaque du support.

4. Conclusion. - La connaissance du mécanisme réactionnel conduisant à la formation du carbure de silicium est de toute première importance pour la métallurgie du silicium. La réactivité du carbone vis-à-vis du silicium fondu dépend de sa microtexture. Si le carbone offre au silicium fondu une enveloppe de couches graphitiques (001) ayant très peu de bords qui émergent, la réactivité chimique est nulle et on observe par endroits que le silicium est directement en contact avec le carbone sans avoir réagi. Dans ce cas, le carbure de silicium qui est toujours présent dans le silicium est en orientation cristallographique rigoureuse avec lui et non avec le carbone : les feuillets de silicium du carbure sont parallèles aux plans (111) du silicium. Il est vraisemblable que dans ce cas, le carbure provient d'une saturation du silicium par le carbone peut-être due à la réaction de l'oxyde de carbone $\mathrm{CO}$ sur le silicium. Le CO est, en effet, toujours présent dans un four où sont chauffées des pièces en graphite. Le papyex, dont la surface spécifique est de l'ordre de plusieurs mètres carrés par gramme, est un réservoir à oxygène et à ce titre une source abondante de $\mathrm{CO}$.

Travail effectué dans le cadre d'un contrat du COMES (Commissariat à l'Energie Solaire). Décision 78-013. 


\section{Bibliographie}

[1] Brissot, J. J., Belouet, C., Cellules solaires au silicium polycristallin, COMPLES Intern. Meeting, Dharan (2-6 nov. 1975). Revue Int. d'Héliotechnique $2^{\mathrm{e}}$ semestre (1975), p. 92-97.

[2] Belouet, C., Brissot, J. J., Martres, R., Croissance des couches de silicium polycristallines sur substrat en carbone pour applications aux cellules solaires. Proc. Col. Int. sur l'Electricité Solaire. Toulouse (1976), p. 191-198.
[3] Belouet, C., Brissot, J. J., Martres, R., Ngo Tich Phuoc, Continuous polycrystalline silicon layers on carbon substrates. Proc. Photovoltaic Solar Energy Conference, Luxembourg (1977) p. 164-175.

[4] Fabre, E., Baudet, Y., Polycrystalline Silicon Solar Cells. Proc. Photovoltaic Solar Energy Conference, Luxembourg (1977) p. 249-258. 